\title{
Homogeneous Plurisubharmonic Polynomials in Higher Dimensions
}

\author{
Lars Simon ${ }^{1}$
}

Received: 11 March 2021 / Accepted: 9 May 2021 / Published online: 21 June 2021

(c) The Author(s) 2021

\section{Abstract}

We prove several results on homogeneous plurisubharmonic polynomials on $\mathbb{C}^{n}$, $n \in \mathbb{Z}_{\geq 2}$. Said results are relevant to the problem of constructing local bumpings at boundary points of pseudoconvex domains of finite D'Angelo 1-type in $\mathbb{C}^{n+1}$.

Keywords Bumping · Finite-type domain · Plurisubharmonic polynomial · Homogeneous polynomial

Mathematics Subject Classification Primary 32U05 · Secondary 32T25

\section{Introduction}

Local bumpings at boundary points of certain bounded, smoothly bounded pseudoconvex domains of finite D'Angelo 1-type in $\mathbb{C}^{n+1}, n \in \mathbb{Z}_{\geq 1}$, have been used both in the construction of peak functions (e.g. [1,5,8]) and in the construction of integral kernels for solving the $\bar{\partial}$-equation (e.g. $[4,9])$.

As explained in $[2,3]$, the problem of constructing such local bumpings naturally leads to the study of homogeneous plurisubharmonic polynomials on $\mathbb{C}^{n}$. Furthermore, in [7], results on homogeneous plurisubharmonic polynomials on $\mathbb{C}^{2}$ by Bharali, Stensønes [3], applied in combination with results from [6], played an important role in establishing sup-norm estimates for solutions to the $\bar{\partial}$-equation for a large class of pseudoconvex domains in $\mathbb{C}^{3}$. Specifically, the crucial results on homogeneous plurisubharmonic polynomials on $\mathbb{C}^{2}$ are the following:

Result ([3, Proposition 1]) Let $P$ be a homogeneous, plurisubharmonic, nonpluriharmonic polynomial on $\mathbb{C}^{2}$. Then there are at most finitely many complex lines through the origin in $\mathbb{C}^{2}$ along which $P$ is harmonic.

$\bowtie \quad$ Lars Simon

lars.simon@ntnu.no; larsimon@gmail.com

1 Department of Mathematical Sciences, Norwegian University of Science and Technology,

Trondheim, Norway 
Result ([3, Theorem 2]) Let $P: \mathbb{C}^{2} \rightarrow \mathbb{R}$ be a non-constant, homogeneous, plurisubharmonic polynomial without pluriharmonic terms. Assume that there exists a holomorphic function $g: \mathbb{C}^{2} \rightarrow \mathbb{C}$, nonsingular on a non-empty open set $U \subseteq \mathbb{C}^{2}$, such that $P$ is harmonic along every level set of $\left.g\right|_{U}$.

Then there exist a homogeneous, subharmonic polynomial $s: \mathbb{C} \rightarrow \mathbb{R}$ and a homogeneous holomorphic polynomial $h: \mathbb{C}^{2} \rightarrow \mathbb{C}$, such that $P=s \circ h$ on $\mathbb{C}^{2}$.

Result ([3, Theorem 3]) Let $P: \mathbb{C}^{2} \rightarrow \mathbb{R}$ be a non-constant, homogeneous, plurisubharmonic polynomial without pluriharmonic terms and assume that $P$ is homogeneous of degree $2 d_{1}$ in $z_{1}, \overline{z_{1}}$ and homogeneous of degree $2 d_{2}$ in $z_{2}, \overline{z_{2}}$, where $d_{1}, d_{2} \in \mathbb{Z}_{\geq 1}$. Then, there exist a homogeneous, subharmonic polynomial $s: \mathbb{C} \rightarrow \mathbb{R}$ without harmonic terms and integers $j, l \in \mathbb{Z}_{\geq 1}$, such that

$$
P\left(z_{1}, z_{2}\right)=s\left(z_{1}^{j} z_{2}^{l}\right) \text { for all }\left(z_{1}, z_{2}\right) \in \mathbb{C}^{2} .
$$

When attempting to adapt the methods from [7] to higher dimensions, it is natural to ask for generalizations of the above-mentioned results on homogeneous plurisubharmonic polynomials on $\mathbb{C}^{2}$ to higher dimensions. Specifically, it is natural to ask the following questions:

Question A Given a homogeneous, plurisubharmonic, non-pluriharmonic polynomial $P$ on $\mathbb{C}^{n}, n \geq 2$, is it true that there are at most finitely many complex hyperplanes through the origin in $\mathbb{C}^{n}$ along which $P$ is pluriharmonic?

Question B Let $P: \mathbb{C}^{n} \rightarrow \mathbb{R}, n \in \mathbb{Z}_{\geq 2}$, be a non-constant, homogeneous, plurisubharmonic polynomial without pluriharmonic terms. Assume that there exists a holomorphic map $G: \mathbb{C}^{n} \rightarrow \mathbb{C}^{m}, 1 \leq m \leq n-1$, nonsingular on a non-empty open set $U \subseteq \mathbb{C}^{n}$, such that $P$ is pluriharmonic along every level set of $\left.G\right|_{U}$.

Are there necessarily a homogeneous, plurisubharmonic polynomial $Q: \mathbb{C}^{m} \rightarrow \mathbb{R}$ and holomorphic polynomials $F_{1}, \ldots, F_{m}: \mathbb{C}^{n} \rightarrow \mathbb{C}$, all homogeneous of the same degree, such that $P=Q \circ\left(F_{1}, \ldots, F_{m}\right)$ on $\mathbb{C}^{n}$ ?

Question C Let $P: \mathbb{C}^{n} \rightarrow \mathbb{R}, n \in \mathbb{Z}_{\geq 2}$, be a non-constant, homogeneous, plurisubharmonic polynomial without pluriharmonic terms and assume $P$ is homogeneous in $l$ variables separately, $1 \leq l \leq n-1$.

Are there necessarily a homogeneous, plurisubharmonic polynomial $Q: \mathbb{C}^{n-l} \rightarrow \mathbb{R}$ and holomorphic polynomials $F_{1}, \ldots, F_{n-l}: \mathbb{C}^{n} \rightarrow \mathbb{C}$, all homogeneous of the same degree, such that $P=Q \circ\left(F_{1}, \ldots, F_{n-l}\right)$ on $\mathbb{C}^{n}$ ?

The purpose of this paper is to provide a detailed answer to Questions A, B and C. A formal statement of the results can be found in Sect. 2.

The answer to Question A is "yes" (Proposition 2.1).

The answer to Question B is "no" in general, even if we additionally assume that the component functions of $G$ are holomorphic polynomials which are all homogeneous of the same degree (Proposition 2.2). However, the answer is "yes" in the special case $m=1$, i.e., when the polynomial is pluriharmonic along the level sets of a single holomorphic function (Theorem 2.3). 
The answer to Question C is "no" in general (Proposition 2.2). However, in the special case where the polynomial is homogeneous in all $n$ variables separately, the answer is "yes" (Corollary 2.5). Furthermore, we get that the answer is "almost yes", or "yes, up to certain singular holomorphic coordinate changes" in the general setting (Theorem 2.4). In many cases, the latter theorem can be used to get the desired bumping results. This is important, since Proposition 2.2 shows that the result by Bharali, Stensønes [3, Theorem 3] does not generalize in this setting.

\section{Statement of Results}

In this section, we state the results of this paper. All the proofs can be found in the later sections.

The answer to Question A is "yes". We have:

Proposition 2.1 Let $P: \mathbb{C}^{n} \rightarrow \mathbb{R}, n \in \mathbb{Z}_{\geq 2}$, be a homogeneous, plurisubharmonic, non-pluriharmonic polynomial. Then there are at most finitely many complex hyperplanes through the origin in $\mathbb{C}^{n}$ along which $P$ is pluriharmonic.

The answer to Question B is "no", even if we additionally assume that the component functions of $G$ are holomorphic polynomials which are all homogeneous of the same degree. The answer to Question C is "no" as well. All of this is implied by the following:

Proposition 2.2 Let $P: \mathbb{C}^{3} \rightarrow \mathbb{R}$,

$$
P\left(z, w_{1}, w_{2}\right)=|z|^{2} \cdot\left(\left|w_{1}\right|^{4}+\left|w_{1}^{2}-w_{1} w_{2}\right|^{2}+\left|w_{2}\right|^{4}\right)
$$

Then $P$ is a non-constant, homogeneous, plurisubharmonic polynomial without pluriharmonic terms and $P$ is homogeneous in one variable separately (see Definition 3.3). Furthermore, away from the coordinate hyperplanes, $P$ is pluriharmonic along the level sets of $G: \mathbb{C}^{3} \rightarrow \mathbb{C}^{2}, G\left(z, w_{1}, w_{2}\right)=\left(z w_{1}^{2}, z w_{2}^{2}\right)$.

However, there do not exist a homogeneous, plurisubharmonic polynomial $Q: \mathbb{C}^{2} \rightarrow$ $\mathbb{R}$ and holomorphic polynomials $F_{1}, F_{2}: \mathbb{C}^{3} \rightarrow \mathbb{C}$, homogeneous of the same degree, such that $P=Q \circ\left(F_{1}, F_{2}\right)$ on $\mathbb{C}^{3}$.

Nevertheless, the answer to Question B in the special case where $m=1$ is "yes":

Theorem 2.3 Let $P: \mathbb{C}^{n} \rightarrow \mathbb{R}, n \in \mathbb{Z}_{\geq 2}$, be a non-constant, homogeneous, plurisubharmonic polynomial without pluriharmonic terms. Assume that there exists a holomorphic function $G: \mathbb{C}^{n} \rightarrow \mathbb{C}$, nonsingular on a non-empty open set $U \subseteq \mathbb{C}^{n}$, such that $P$ is pluriharmonic along every level set of $\left.G\right|_{U}$.

Then, there exist a homogeneous, subharmonic polynomial $s: \mathbb{C} \rightarrow \mathbb{R}$ without harmonic terms and a homogeneous holomorphic polynomial $h: \mathbb{C}^{n} \rightarrow \mathbb{C}$, such that $P=s \circ h$ on $\mathbb{C}^{n}$.

Note that Theorem 2.3 generalizes the upper mentioned result by Bharali, Stensønes [3, Theorem 2] to higher dimension. 
Although the answer to Question C is "no", we get the following result, which says that the answer is "yes, up to singular holomorphic coordinate changes":

Theorem 2.4 Let $P: \mathbb{C}^{n} \rightarrow \mathbb{R}, n \in \mathbb{Z}_{\geq 2}$, be a non-constant plurisubharmonic polynomial without pluriharmonic terms and assume that $P$ is homogeneous of degree $2 k, k \in \mathbb{Z}_{\geq 1}$. Let $1 \leq l \leq n-1$ and assume that $P$ is homogeneous of degree $2 d_{j}$, $d_{j} \in \mathbb{Z}_{>0}$, in $z_{j}, \overline{z_{j}}$ for $j=1, \ldots, l$ (see Definition 3.3). Assume furthermore that $k-D>0$, where $D=d_{1}+\cdots+d_{l}$. Write $d:=\operatorname{gcd}\left(d_{1}, \ldots, d_{l}, k\right) \in \mathbb{Z}_{\geq 1}$.

Then, there exists a plurisubharmonic polynomial $Q: \mathbb{C}^{n-l} \rightarrow \mathbb{R}$ without pluriharmonic terms, homogeneous of degree $2 k-2 D$, with the property that both the holomorphic and the anti-holomorphic degree (see Sect. 3) of every term appearing in $Q$ are divisible by the integer $(k-D) / d$, such that we have for all $\left(z_{1}, \ldots, z_{n}\right) \in \mathbb{C}^{n}$ :

$$
P\left(z_{1}, \ldots, z_{n}\right)=Q\left(\tau z_{l+1}, \ldots, \tau z_{n}\right),
$$

for every solution $\tau \in \mathbb{C}$ of $\tau^{(k-D) / d}=z_{1}^{d_{1} / d} \cdots z_{l}^{d_{l} / d}$.

Alternatively, we can carry out a singular holomorphic coordinate change $\Phi: \mathbb{C}^{n} \rightarrow \mathbb{C}^{n},\left(z_{1}, \ldots, z_{n}\right) \mapsto\left(z_{1}{ }^{(k-D) / d}, \ldots, z_{l}(k-D) / d, z_{l+1}, \ldots, z_{n}\right)$ and write

$$
(P \circ \Phi)\left(z_{1}, \ldots, z_{n}\right)=Q\left(z_{1}^{d_{1} / d} \cdots z_{l}^{d_{l} / d} z_{l+1}, \ldots, z_{1}^{d_{1} / d} \cdots z_{l}^{d_{l} / d} z_{n}\right) .
$$

Note that, without the assumptions $d_{1}, \ldots, d_{l}, k-D>0$ in Theorem $2.4, P$ is effectively a polynomial in fewer than $n$ variables, hence we can ignore that case.

As a corollary (of the proof) of Theorem 2.4 we get that the answer to Question C in the special case where $P$ is homogeneous in all $n$ variables separately is "yes":

Corollary 2.5 Let $P: \mathbb{C}^{n} \rightarrow \mathbb{R}, n \in \mathbb{Z}_{\geq 2}$, be a non-constant, homogeneous, plurisubharmonic polynomial without pluriharmonic terms and assume that $P$ is homogeneous of degree $2 d_{j}, d_{j} \in \mathbb{Z}_{>0}$, in $z_{j}, \overline{z_{j}}$ for $j=1, \ldots, n$ (see Definition 3.3). Write $d:=\operatorname{gcd}\left(d_{1}, \ldots, d_{n}\right) \in \mathbb{Z}_{\geq 1}$.

Then, there exists a homogeneous, subharmonic polynomial $s: \mathbb{C} \rightarrow \mathbb{R}$ without harmonic terms, such that

$$
P\left(z_{1}, \ldots, z_{n}\right)=s\left(z_{1}{ }^{d_{1} / d} \cdots z_{n}{ }^{d_{n} / d}\right)
$$

for all $\left(z_{1}, \ldots, z_{n}\right) \in \mathbb{C}^{n}$.

Note that Corollary 2.5 generalizes the upper mentioned result by Bharali, Stens $\varnothing$ nes [3, Theorem 3] to higher dimension.

\section{Preliminaries}

For the remainder of this section we fix an integer $n \geq 2$ and a non-constant polynomial $P: \mathbb{C}^{n} \rightarrow \mathbb{R}$ with the following properties:

- $P$ is $\mathbb{R}$-homogeneous of degree $2 k$, for some positive integer $k$, 
- $P$ is plurisubharmonic,

- $P$ does not have any pluriharmonic terms (i.e., purely holomorphic or purely antiholomorphic terms).

In particular, there exists a collection $\left(a_{\alpha, \beta}\right)_{(\alpha, \beta) \in \mathcal{J}}$ of complex numbers where

- $\mathcal{J}$ is the set of all pairs $(\alpha, \beta) \in\left(\mathbb{Z}_{\geq 0}\right)^{n} \times\left(\mathbb{Z}_{\geq 0}\right)^{n}$ satisfying $|\alpha|>0,|\beta|>0$ and $|\alpha|+|\beta|=2 k$,

- $\overline{a_{\alpha, \beta}}=a_{\beta, \alpha}$ for all $(\alpha, \beta) \in \mathcal{J}$,

such that

$$
P(z)=\sum_{(\alpha, \beta) \in \mathcal{J}} a_{\alpha, \beta} z^{\alpha} \bar{z}^{\beta}
$$

for all $z=\left(z_{1}, \ldots, z_{n}\right) \in \mathbb{C}^{n}$. Here we are making use of the usual multi-index notation: $|\alpha|=\alpha_{1}+\cdots+\alpha_{n}$ and $z^{\alpha}=z_{1}^{\alpha_{1}} \cdots z_{n}^{\alpha_{n}}$ (and analogously for $\beta$ and $\bar{z}^{\beta}$ ). If $a_{\alpha, \beta} \neq 0$, then we say that $|\alpha|$ (resp. $\left.|\beta|\right)$ is the holomorphic (resp. anti-holomorphic) degree of the term $a_{\alpha, \beta} z^{\alpha} \bar{z}^{\beta}$.

Furthermore, let $\mathcal{L}(P ; p, V)$ denote the Levi form of $P$ at the point $p \in \mathbb{C}^{n}$ in direction $V=\left(V_{1}, \ldots, V_{n}\right)^{t} \in \mathbb{C}^{n}$, i.e.,

$$
\mathcal{L}(P ; p, V)=\left(V_{1}, \ldots, V_{n}\right)\left(\begin{array}{ccc}
\frac{\partial^{2} P}{\partial z_{1} \partial \overline{z_{1}}}(p) & \ldots & \frac{\partial^{2} P}{\partial z_{1} \partial \overline{z_{n}}}(p) \\
\vdots & \ddots & \vdots \\
\frac{\partial^{2} P}{\partial z_{n} \partial \overline{z_{1}}}(p) & \ldots & \frac{\partial^{2} P}{\partial z_{n} \partial \overline{z_{n}}}(p)
\end{array}\right)\left(\begin{array}{c}
\overline{V_{1}} \\
\vdots \\
\overline{V_{n}}
\end{array}\right) .
$$

Lemma 3.1 Let $\mathcal{A}=\left\{\alpha \in\left(\mathbb{Z}_{\geq 0}\right)^{n}:|\alpha|=k\right.$ and $\left.a_{\alpha, \alpha} \neq 0\right\}$ and let

$$
\mathcal{C}=\left\{\left(c_{1}, \ldots, c_{n}\right) \in \mathbb{C}^{n}: \sum_{j=1}^{n} \alpha_{j} c_{j}=0 \text { for all } \alpha \in \mathcal{A}\right\}
$$

Then we have for all $\left(c_{1}, \ldots, c_{n}\right) \in \mathcal{C}$ and for all $z=\left(z_{1}, \ldots, z_{n}\right) \in \mathbb{C}^{n}$ :

$$
\mathcal{L}\left(P ; z,\left(c_{1} z_{1}, \ldots, c_{n} z_{n}\right)^{t}\right)=0 .
$$

Proof For all $z=\left(z_{1}, \ldots, z_{n}\right) \in \mathbb{C}^{n},\left(c_{1}, \ldots, c_{n}\right) \in \mathbb{C}^{n}$ a straightforward calculation shows that

$$
\mathcal{L}\left(P ; z,\left(c_{1} z_{1}, \ldots, c_{n} z_{n}\right)^{t}\right)=\sum_{(\alpha, \beta) \in \mathcal{J}} a_{\alpha, \beta} \cdot\left(\sum_{j=1}^{n} \alpha_{j} c_{j}\right) \cdot \overline{\left(\sum_{j=1}^{n} \beta_{j} c_{j}\right)} \cdot z^{\alpha} \bar{z}^{\beta} .
$$

Assume for the sake of a contradiction that the claim is wrong. We then find some $\left(c_{1}, \ldots, c_{n}\right) \in \mathcal{C}$ and some $r_{1}, \ldots, r_{n} \in \mathbb{R}_{\geq 0}, \phi_{1}, \ldots, \phi_{n} \in[0,2 \pi)$, such that:

$$
\mathcal{L}\left(P ;\left(r_{1} e^{i \phi_{1}}, \ldots, r_{n} e^{i \phi_{n}}\right),\left(c_{1} r_{1} e^{i \phi_{1}}, \ldots, c_{n} r_{n} e^{i \phi_{n}}\right)^{t}\right) \neq 0
$$


By continuity, and since $P$ is plurisubharmonic, we then get:

$$
\begin{aligned}
0< & \int_{0}^{2 \pi} \ldots \int_{0}^{2 \pi} \mathcal{L}\left(P ;\left(r_{1} e^{i \theta_{1}}, \ldots, r_{n} e^{i \theta_{n}}\right),\left(c_{1} r_{1} e^{i \theta_{1}}, \ldots, c_{n} r_{n} e^{i \theta_{n}}\right)^{t}\right) d \theta_{1} \ldots d \theta_{n} \\
= & \sum_{(\alpha, \beta) \in \mathcal{J}} a_{\alpha, \beta} \cdot\left(\sum_{j=1}^{n} \alpha_{j} c_{j}\right) \cdot \overline{\left(\sum_{j=1}^{n} \beta_{j} c_{j}\right)} \cdot r_{1}{ }^{\alpha_{1}+\beta_{1}} \ldots r_{n}{ }^{\alpha_{n}+\beta_{n}} \\
& \cdot\left(\int_{0}^{2 \pi} e^{i\left(\alpha_{1}-\beta_{1}\right) \theta_{1}} d \theta_{1}\right) \ldots\left(\int_{0}^{2 \pi} e^{i\left(\alpha_{n}-\beta_{n}\right) \theta_{n}} d \theta_{n}\right) \\
= & (2 \pi)^{n} \sum_{\alpha \in \mathcal{A}} a_{\alpha, \alpha} \cdot\left|\sum_{j=1}^{n} \alpha_{j} c_{j}\right|^{2} \cdot r_{1}^{\alpha_{1}+\alpha_{1}} \ldots r_{n}{ }^{\alpha_{n}+\alpha_{n}} \\
= & 0,
\end{aligned}
$$

where the last equality is due to the fact that $\left(c_{1}, \ldots, c_{n}\right) \in \mathcal{C}$. We have arrived at the desired contradiction; the claim follows.

For all $\beta \in\left(\mathbb{Z}_{\geq 0}\right)^{n}$ with $1 \leq|\beta| \leq 2 k-1$ we define a homogeneous holomorphic polynomial $P_{\beta}: \mathbb{C}^{n} \rightarrow \mathbb{C}$,

$$
P_{\beta}(z)=\sum_{\alpha:|\alpha|=2 k-|\beta|} a_{\alpha, \beta} z^{\alpha} .
$$

In particular, we can write

$$
P(z)=\sum_{\beta: 1 \leq|\beta| \leq 2 k-1} \bar{z}^{\beta} P_{\beta}(z) .
$$

Lemma 3.2 Assume that there exists a holomorphic map $G: \mathbb{C}^{n} \rightarrow \mathbb{C}^{m}, 1 \leq m \leq$ $n-1$, nonsingular on a non-empty open set $U \subseteq \mathbb{C}^{n}$, such that $P$ is pluriharmonic along every level set of $\left.G\right|_{U}$.

Then, for all $i_{1}, \ldots, i_{m}, L \in\{1, \ldots, n\}$ (not necessarily pairwise distinct) and for all $\beta \in\left(\mathbb{Z}_{\geq 0}\right)^{n}$ with $1 \leq|\beta| \leq 2 k-1$, the following equality holds on $\mathbb{C}^{n}$ :

$$
\operatorname{det}\left(\begin{array}{ccc}
\frac{\partial G_{1}}{\partial z_{i_{1}}} & \cdots & \frac{\partial G_{1}}{\partial z_{i_{m}}} \\
\vdots & \ddots & \vdots \\
\frac{\partial G_{m}}{\partial z_{i_{1}}} & \cdots & \frac{\partial G_{m}}{\partial z_{i_{m}}}
\end{array}\right) \cdot \frac{\partial P_{\beta}}{\partial z_{L}}=\sum_{j=1}^{m} \operatorname{det}\left(\begin{array}{ccc}
\frac{\partial G_{1}}{\partial z_{i_{1}}} & \cdots & \frac{\partial G_{1}}{\partial z_{i_{m}}} \\
\vdots & \ddots & \vdots \\
\frac{\partial G_{j-1}}{\partial z_{i_{1}}} & \cdots & \frac{\partial G_{j-1}}{\partial z_{i_{m}}} \\
\frac{\partial P_{\beta}}{\partial z_{i_{1}}} & \cdots & \frac{\partial P_{\beta}}{\partial z_{i_{m}}} \\
\frac{\partial G_{j+1}}{\partial z_{i_{1}}} & \cdots & \frac{\partial G_{j+1}}{\partial z_{i_{m}}} \\
\vdots & \ddots & \vdots \\
\frac{\partial G_{m}}{\partial z_{i_{1}}} & \cdots & \frac{\partial G_{m}}{\partial z_{i_{m}}}
\end{array}\right) \cdot \frac{\partial G_{j}}{\partial z_{L}}
$$


Proof Fix a point $p \in U$. Then there exist an open neighborhood $W \subseteq U$ of $p$ and holomorphic maps $K_{1}, \ldots, K_{n-m}: W \rightarrow \mathbb{C}^{n} \backslash\{0\}$, such that $\left\{K_{1}(z), \ldots, K_{n-m}(z)\right\}$ is a basis for the null space of $G^{\prime}(z) \in \mathbb{C}^{m \times n}$ for all $z \in W$. For $l=1, \ldots, n-m$, denote the component functions of $K_{l}$ as $K_{l}^{(1)}, \ldots, K_{l}{ }^{(n)}$. Since $P$ is pluriharmonic along every level set of $\left.G\right|_{U}$, we get

$$
0=\left(K_{l}^{(1)}, \ldots, K_{l}^{(n)}\right)\left(\begin{array}{ccc}
\frac{\partial^{2} P}{\partial z_{1} \partial \overline{z_{1}}} & \cdots & \frac{\partial^{2} P}{\partial z_{1} \partial \overline{z_{n}}} \\
\vdots & \ddots & \vdots \\
\frac{\partial^{2} P}{\partial z_{n} \partial \overline{z_{1}}} & \cdots & \frac{\partial^{2} P}{\partial z_{n} \partial \overline{z_{n}}}
\end{array}\right)\left(\begin{array}{c}
\overline{K_{l}^{(1)}} \\
\vdots \\
\overline{K_{l}^{(n)}}
\end{array}\right)
$$

on $W$ for $l=1, \ldots, n-m$; but since the Complex Hessian matrix of $P$ is positive semidefinite, we even get

$$
(0, \ldots, 0)=\left(K_{l}^{(1)}, \ldots, K_{l}^{(n)}\right)\left(\begin{array}{ccc}
\frac{\partial^{2} P}{\partial z_{1} \partial \overline{z_{1}}} & \cdots & \frac{\partial^{2} P}{\partial z_{1} \partial \overline{z_{n}}} \\
\vdots & \ddots & \vdots \\
\frac{\partial^{2} P}{\partial z_{n} \partial \overline{z_{1}}} & \cdots & \frac{\partial^{2} P}{\partial z_{n} \partial \overline{z_{n}}}
\end{array}\right)
$$

Writing

$$
P(z)=\sum_{\beta: 1 \leq|\beta| \leq 2 k-1} \bar{z}^{\beta} P_{\beta}(z),
$$

as above, we get for $I=1, \ldots, n$ :

$$
\begin{aligned}
0 & =\sum_{J=1}^{n} K_{l}^{(J)}(z) \cdot \frac{\partial^{2} P}{\partial z_{J} \partial \overline{z_{I}}}(z) \\
& =\sum_{\beta: 1 \leq|\beta| \leq 2 k-1}\left(\beta_{I} \overline{z_{1}} \beta_{1} \ldots \overline{z_{I}} \beta_{I}-1 \ldots \overline{z_{n}} \beta_{n} \cdot \sum_{J=1}^{n} K_{l}^{(J)}(z) \frac{\partial P_{\beta}}{\partial z_{J}}(z)\right)
\end{aligned}
$$

for all $z \in W, l=1, \ldots, n-m$; hence

$$
\begin{aligned}
0 & =\sum_{I=1}^{n} \overline{z_{I}} \cdot 0 \\
& =\sum_{\beta: 1 \leq|\beta| \leq 2 k-1}\left(|\beta| \cdot \bar{z}^{\beta} \cdot \sum_{J=1}^{n} K_{l}^{(J)}(z) \frac{\partial P_{\beta}}{\partial z_{J}}(z)\right) .
\end{aligned}
$$


Owing to the fact that the $K_{l}^{(J)}$ and the $P_{\beta}$ are holomorphic, we then get for all $\beta \in\left(\mathbb{Z}_{\geq 0}\right)^{n}$ with $1 \leq|\beta| \leq 2 k-1$ :

$$
0=\sum_{J=1}^{n} K_{l}^{(J)}(z) \frac{\partial P_{\beta}}{\partial z_{J}}(z) \text { for all } z \in W, l \in\{1, \ldots, n-m\},
$$

i.e., $K_{l}(z)=\left(K_{l}^{(1)}(z), \ldots, K_{l}^{(n)}(z)\right)^{t}$ is in the null space of the matrix $P_{\beta}^{\prime}(z) \in \mathbb{C}^{1 \times n}$. But this implies that the null space of the matrix

$$
\left(\begin{array}{ccc}
\frac{\partial G_{1}}{\partial z_{1}}(z) & \cdots & \frac{\partial G_{1}}{\partial z_{n}}(z) \\
\vdots & \ddots & \vdots \\
\frac{\partial G_{m}}{\partial z_{1}}(z) & \cdots & \frac{\partial G_{m}}{\partial z_{n}}(z) \\
\frac{\partial P_{\beta}}{\partial z_{1}}(z) & \cdots & \frac{\partial P_{\beta}}{\partial z_{n}}(z)
\end{array}\right) \in \mathbb{C}^{(m+1) \times n}, z \in W,
$$

is $(n-m)$-dimensional for all $\beta$ with $1 \leq|\beta| \leq 2 k-1$, so the rank of said matrix is $m$. Hence, given $i_{1}, \ldots, i_{m}, L \in\{1, \ldots, n\}$, we have

$$
0=\operatorname{det}\left(\begin{array}{cccc}
\frac{\partial G_{1}}{\partial z_{1}} & \cdots & \frac{\partial G_{1}}{\partial z_{i_{m}}} & \frac{\partial G_{1}}{\partial z_{L}} \\
\vdots & \ddots & \vdots & \vdots \\
\frac{\partial G_{m}}{\partial z_{i_{1}}} & \cdots & \frac{\partial G_{m}}{\partial z_{m}} & \frac{\partial G_{m}}{\partial z_{L}} \\
\frac{\partial P_{\beta}}{\partial z_{i_{1}}} & \cdots & \frac{\partial P_{\beta}}{\partial z_{i_{m}}} & \frac{\partial P_{\beta}}{\partial z_{L}}
\end{array}\right)
$$

on $W$ for all $\beta$ with $1 \leq|\beta| \leq 2 k-1$; noting that all the entries of the latter matrix are holomorphic on $\mathbb{C}^{n}$, the identity theorem gives that the determinant vanishes on all of $\mathbb{C}^{n}$. The claim follows by Laplace expanding by the last column and calculating.

Definition 3.3 Given $l \in\{1, \ldots, n\}$, we say that $P$ is homogeneous in $l$ variables separately, provided there exist integers $1 \leq i_{1}<\cdots<i_{l} \leq n$ and integers $d_{i_{1}}, \ldots, d_{i_{l}} \geq 1$, such that for every $(\alpha, \beta) \in \mathcal{J}$ with $a_{\alpha, \beta} \neq 0$ we have $\alpha_{i_{1}}+\beta_{i_{1}}=2 d_{i_{1}}, \ldots, \alpha_{i_{l}}+\beta_{i_{l}}=2 d_{i_{l}}$. In this case we say that $P$ is homogeneous of degree $2 d_{i_{j}}$ in $z_{i_{j}}, \overline{z_{i_{j}}}$ for all $j \in\{1, \ldots, l\}$.

Note 3.4 We restrict attention to even degrees in Definition 3.3 due to the plurisubharmonicity requirement. Note furthermore that, in the case $l=n-1$, the polynomial $P$ is necessarily homogeneous in all $n$ variables separately, since $P$ is homogeneous.

Lemma 3.5 Let $1 \leq l \leq n-1$ and assume that $P$ is homogeneous of degree $2 d_{j}$, $d_{j} \in \mathbb{Z}_{>0}$, in $z_{j}$, $\overline{z_{j}}$ for $j=1, \ldots, l$. Assume furthermore that $k-D>0$, where $D=d_{1}+\cdots+d_{l}$.

Then, away from the coordinate hyperplanes, $P$ is pluriharmonic along the level sets of $G: \mathbb{C}^{n} \rightarrow \mathbb{C}^{n-l}$,

$$
G\left(z_{1}, \ldots, z_{n}\right)=z_{1}{ }^{d_{1}} \cdots z_{l}{ }^{d_{l}} \cdot\left(z_{l+1}{ }^{k-D}, z_{l+2}{ }^{k-D}, \ldots, z_{n}{ }^{k-D}\right) .
$$


Proof $G$ is nonsingular on $U:=\left\{\left(z_{1}, \ldots, z_{n}\right) \in \mathbb{C}^{n}: z_{1} \neq 0, \ldots, z_{n} \neq 0\right\}$. Given $z \in U$, we have to show that $\mathcal{L}(P ; z, V)=0$ for all $V$ in the null space of $G^{\prime}(z) \in$ $\mathbb{C}^{(n-l) \times n}$. But since the Complex Hessian matrix of $P$ is positive semidefinite, it suffices to verify this for a basis of said null space. If $z \in U$, then the collection of vectors

$$
\left(\begin{array}{c}
0 \\
\vdots \\
0 \\
(k-D) z_{j} \\
0 \\
\vdots \\
0 \\
-d_{j} z_{l+1} \\
\vdots \\
-d_{j} z_{n}
\end{array}\right) \quad \text {, where } j \in\{1, \ldots, l\}
$$

forms a basis for the null space of $G^{\prime}(z) \in \mathbb{C}^{(n-l) \times n}$. Hence, with $\mathcal{A}$ and $\mathcal{C}$ as in Lemma 3.1, it suffices to show that $C_{j} \in \mathcal{C}$ for $j \in\{1, \ldots, l\}$, where

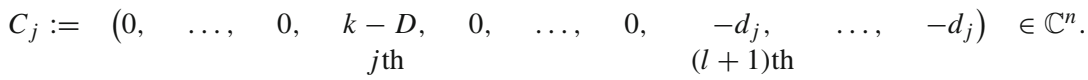

To this end, we consider some $j \in\{1, \ldots, l\}$ and some $\alpha \in \mathcal{A}$. Since $a_{\alpha, \alpha} \neq 0$ and by assumption on $P$ we then have $\alpha_{1}=d_{1}, \ldots, \alpha_{l}=d_{l}$, and $\alpha_{l+1}+\cdots+\alpha_{n}=k-D$. Writing $C_{j}=:\left(c_{1}{ }^{(j)}, \ldots, c_{n}{ }^{(j)}\right)$, we then have:

$$
\sum_{s=1}^{n} \alpha_{s} c_{s}^{(j)}=(k-D) \alpha_{j}-d_{j}\left(\alpha_{l+1}+\cdots+\alpha_{n}\right)=0
$$

as desired.

\section{Proof of Proposition 2.1}

Proof of Proposition 2.1 We proceed by induction on the dimension $n$. The 2dimensional case was handled by Bharali, Stensønes [3], so let $n \geq 3$ and assume the claim holds in dimensions $2, \ldots, n-1$. Let $P$ be as in the statement of the theorem and assume for the sake of a contradiction that $P$ is pluriharmonic along infinitely many complex hyperplanes through $0 \in \mathbb{C}^{n}$. We then find a sequence $\left(\mathcal{H}_{j}\right)_{j \in \mathbb{Z} \geq 1}$ of pairwise distinct such hyperplanes. It is furthermore easy to see that there exists a complex hyperplane $A$ through $0 \in \mathbb{C}^{n}$, such that $P$ is not pluriharmonic along $A$. Since $P$ is pluriharmonic along each $\mathcal{H}_{j}, j \in \mathbb{Z}_{\geq 1}$, we get that $P$ is pluriharmonic 
along $A \cap \mathcal{H}_{j}$ for all $j \in \mathbb{Z}_{\geq 1}$. Hence, by induction, the set $\left\{A \cap \mathcal{H}_{j}: j \in \mathbb{Z}_{\geq 1}\right\}$ is finite. So, there exists an $(n-2)$-dimensional complex vector subspace $V$ of $A$, such that $A \cap \mathcal{H}_{j}=V$ for infinitely many $j \in \mathbb{Z}_{\geq 1}$. Thus, after deleting some members of the sequence if necessary, we can assume that

$$
A \cap \mathcal{H}_{j}=V \text { for all } j \in \mathbb{Z}_{\geq 1}
$$

It is easy to verify that there exists a complex hyperplane $B$ through $0 \in \mathbb{C}^{n}$, such that $P$ is not pluriharmonic along $B$ and $B$ does not contain $V$. By repeating the same argument and again deleting some members of the sequence if necessary, we find an $(n-2)$-dimensional complex vector subspace $W$ of $B$, such that

$$
B \cap \mathcal{H}_{j}=W \text { for all } j \in \mathbb{Z}_{\geq 1}
$$

Hence every $\mathcal{H}_{j}$ contains $V+W$. However, $B$ contains $W$ but does not contain $V$, so we get that $V+W$ is at least $(n-1)$-dimensional. We conclude that $\mathcal{H}_{j}=V+W$ for all $j \in \mathbb{Z}_{\geq 1}$. Since the members of the sequence $\left(\mathcal{H}_{j}\right)_{j \in \mathbb{Z}_{\geq 1}}$ were chosen to be pairwise distinct, we have arrived at the desired contradiction.

\section{Proof of Proposition 2.2}

Let $P$ and $G$ be as in the statement of Proposition 2.2. It is obvious that $P$ is indeed a non-constant, homogeneous, plurisubharmonic polynomial without pluriharmonic terms and that $P$ is homogeneous of degree 2 in $z, \bar{z}$, so $P$ is homogeneous in one variable separately. Furthermore, away from the coordinate hyperplanes, $P$ is pluriharmonic along the level sets of $G$ by Lemma 3.5.

Assume for the sake of a contradiction that there exist a homogeneous, plurisubharmonic polynomial $Q: \mathbb{C}^{2} \rightarrow \mathbb{R}$ and holomorphic polynomials $F_{1}, F_{2}: \mathbb{C}^{3} \rightarrow \mathbb{C}$, homogeneous of the same degree, such that $P=Q \circ\left(F_{1}, F_{2}\right)$ on $\mathbb{C}^{3}$. As in Sect. 3 we write

$$
\begin{aligned}
P\left(z, w_{1}, w_{2}\right)= & \bar{z} \cdot \bar{w}_{1}^{2} \cdot\left(2 z w_{1}^{2}-z w_{1} w_{2}\right)+\bar{z} \cdot \overline{w_{1}} \cdot \overline{w_{2}} \cdot\left(z w_{1} w_{2}-z w_{1}^{2}\right) \\
& +\bar{z} \cdot \bar{w}_{2}^{2} \cdot\left(z w_{2}^{2}\right) .
\end{aligned}
$$

Even though the holomorphic map $\left(F_{1}, F_{2}\right): \mathbb{C}^{3} \rightarrow \mathbb{C}^{2}$ is (a priori) not necessarily non-singular, an argument analogous to the proof of Lemma 3.2 gives that the matrix

$$
\left(\begin{array}{ccc}
\frac{\partial F_{1}}{\partial z} & \frac{\partial F_{1}}{\partial w_{1}} & \frac{\partial F_{1}}{\partial w_{2}} \\
\frac{\partial F_{2}}{\partial z} & \frac{\partial F_{2}}{\partial w_{1}} & \frac{\partial F_{2}}{\partial w_{2}} \\
2 w_{1}^{2}-w_{1} w_{2} & 4 z w_{1}-z w_{2} & -z w_{1} \\
w_{1} w_{2}-w_{1}^{2} & -2 z w_{1}+z w_{2} & z w_{1} \\
w_{2}^{2} & 0 & 2 z w_{2}
\end{array}\right)
$$


has the same rank as the matrix

$$
\left(\begin{array}{lll}
\frac{\partial F_{1}}{\partial z} & \frac{\partial F_{1}}{\partial w_{1}} & \frac{\partial F_{1}}{\partial w_{2}} \\
\frac{\partial F_{2}}{\partial z} & \frac{\partial F_{2}}{\partial w_{1}} & \frac{\partial F_{2}}{\partial w_{2}}
\end{array}\right)
$$

at every point of some non-empty open subset $U$ of $\mathbb{C}^{3}$, which does not meet the coordinate hyperplanes. In particular, said rank is 2 and $\left(F_{1}, F_{2}\right)$ is non-singular on $U$; hence $F_{1}$ and $F_{2}$ are both non-constant and homogeneous of degree $d:=\operatorname{deg} F_{1}=$ $\operatorname{deg} F_{2} \geq 1$. After adding the fourth row (of the former matrix) to the third row and applying the identity theorem, we get that the following holds on $\mathbb{C}^{3}$ for $j \in\{1,2\}$ :

$$
0=\operatorname{det}\left(\begin{array}{ccc}
\frac{\partial F_{j}}{\partial z} & \frac{\partial F_{j}}{\partial w_{1}} & \frac{\partial F_{j}}{\partial w_{2}} \\
w_{1}^{2} & 2 z w_{1} & 0 \\
w_{2}^{2} & 0 & 2 z w_{2}
\end{array}\right)
$$

and, using that $F_{j}$ is homogeneous, a calculation then gives

$$
3 z \frac{\partial F_{j}}{\partial z}=z \frac{\partial F_{j}}{\partial z}+w_{1} \frac{\partial F_{j}}{\partial w_{1}}+w_{2} \frac{\partial F_{j}}{\partial w_{2}}=d \cdot F_{j}
$$

From this we readily deduce that $d / 3$ is a positive integer and that there exist holomorphic polynomials $0 \not \equiv f_{1}, f_{2}: \mathbb{C}^{2} \rightarrow \mathbb{C}$, homogeneous of degree $2 d / 3$, such that

$$
F_{j}\left(z, w_{1}, w_{2}\right)=z^{\frac{d}{3}} \cdot f_{j}\left(w_{1}, w_{2}\right) \text { on } \mathbb{C}^{3}
$$

for $j \in\{1,2\}$. Since $Q: \mathbb{C}^{2} \rightarrow \mathbb{R}$ is a homogeneous, plurisubharmonic (and clearly also non-pluriharmonic) polynomial, its degree $\operatorname{deg} Q$ is even. But $d / 3$ is an integer and $6=\operatorname{deg} P=d \cdot \operatorname{deg} Q$, so we necessarily have $\operatorname{deg} Q=2$ and $d=3$. In particular, $f_{j}$ is homogeneous of degree 2 and $F_{j}\left(z, w_{1}, w_{2}\right)=z \cdot f_{j}\left(w_{1}, w_{2}\right)$ on $\mathbb{C}^{3}$ for $j \in\{1,2\}$. Since $P$ does not have any pluriharmonic terms, we can assume that $Q$ does not have any pluriharmonic terms either. So there exist $a, c \in \mathbb{R}, b \in \mathbb{C}$, such that we have for all $(x, y) \in \mathbb{C}^{2}$ :

$$
Q(x, y)=a \cdot|x|^{2}+b \cdot x \bar{y}+\bar{b} \cdot \bar{x} y+c \cdot|y|^{2} .
$$

For $j \in\{1,2\}$ we furthermore find $\sigma_{j}, \rho_{j}, \mu_{j} \in \mathbb{C}$, such that we have for all $\left(w_{1}, w_{2}\right) \in$ $\mathbb{C}^{2}$ :

$$
f_{j}\left(w_{1}, w_{2}\right)=\sigma_{j} w_{1}^{2}+\rho_{j} w_{1} w_{2}+\mu_{j} w_{2}^{2}
$$

A calculation then shows that 


$$
\begin{aligned}
& Q\left(z \cdot f_{1}\left(w_{1}, w_{2}\right), z \cdot f_{2}\left(w_{1}, w_{2}\right)\right) \\
& \quad=|z|^{2} \cdot\left({\overline{w_{1}}}^{2} \cdot g_{1}\left(w_{1}, w_{2}\right)+\overline{w_{1} w_{2}} \cdot h\left(w_{1}, w_{2}\right)+{\overline{w_{2}}}^{2} \cdot g_{2}\left(w_{1}, w_{2}\right)\right)
\end{aligned}
$$

for some $g_{1}, h, g_{2}: \mathbb{C}^{2} \rightarrow \mathbb{C}$ contained in the $\mathbb{C}$-vector space $\mathcal{V}$ spanned by $f_{1}$ and $f_{2}$; we trivially have $\operatorname{dim}_{\mathbb{C}} \mathcal{V} \leq 2$. Recalling that $P=Q \circ\left(F_{1}, F_{2}\right)$, we necessarily have

$$
g_{1}\left(w_{1}, w_{2}\right)=2 w_{1}^{2}-w_{1} w_{2}, \quad h\left(w_{1}, w_{2}\right)=w_{1} w_{2}-w_{1}^{2}, \quad g_{2}\left(w_{1}, w_{2}\right)=w_{2}^{2}
$$

which implies that $\operatorname{dim}_{\mathbb{C}} \mathcal{V} \geq 3$. We have arrived at the desired contradiction.

\section{Proof of Theorem 2.3}

In this section we will (without further comment) identify holomorphic polynomials $\mathbb{C}^{n} \rightarrow \mathbb{C}$ with elements of the polynomial ring $\mathbb{C}\left[z_{1}, \ldots, z_{n}\right]$ in the obvious way.

Notation 6.1 Let $0 \neq g \in \mathbb{C}\left[z_{1}, \ldots, z_{n}\right]$ be a homogeneous polynomial of positive degree. Then the set

$$
\left\{m \in \mathbb{Z}_{>0}: g=\widetilde{g}^{m} \text { for some homogeneous } \tilde{g} \in \mathbb{C}\left[z_{1}, \ldots, z_{n}\right]\right\}
$$

is clearly non-empty and bounded from above. Hence it has a maximum, which we denote as $M_{g} \in \mathbb{Z}_{>0}$.

Lemma 6.2 Let $0 \neq g \in \mathbb{C}\left[z_{1}, \ldots, z_{n}\right]$ be a homogeneous polynomial of positive degree and let $h \in \mathbb{C}\left[z_{1}, \ldots, z_{n}\right]$ be any homogeneous polynomial with $g=h^{M_{g}}$ (see Notation 6.1). If $0 \neq f \in \mathbb{C}\left[z_{1}, \ldots, z_{n}\right]$ is a homogeneous polynomial of positive degree satisfying

$$
(\operatorname{deg} g) \cdot g \cdot \frac{\partial f}{\partial z_{l}}=(\operatorname{deg} f) \cdot f \cdot \frac{\partial g}{\partial z_{l}} \text { for all } l \in\{1, \ldots, n\},
$$

then $M_{g} \cdot(\operatorname{deg} f) /(\operatorname{deg} g)$ is a positive integer and there exists a $c \in \mathbb{C} \backslash\{0\}$, such that $f=c \cdot h^{M_{g} \cdot \frac{\operatorname{deg} f}{\operatorname{deg} g}}$.

Proof Let $g, h, f \in \mathbb{C}\left[z_{1}, \ldots, z_{n}\right]$ be as in the statement of the lemma. Write

$$
g=u p_{1}{ }^{\alpha_{1}} \cdots p_{m}{ }^{\alpha_{m}}
$$

where $m$ is a positive integer (since $\operatorname{deg} g>0$ ), $u \in \mathbb{C} \backslash\{0\}$ is a unit, $p_{1}, \ldots, p_{m} \in$ $\mathbb{C}\left[z_{1}, \ldots, z_{n}\right]$ are pairwise non-associate primes, and $\alpha_{1}, \ldots, \alpha_{m}$ are positive integers. Since $\operatorname{deg} f>0$, we get that $g$ divides $f \cdot(\partial g) /\left(\partial z_{l}\right)$ for $l=1, \ldots, n$. Hence, considering any $s \in\{1, \ldots, m\}$, we get that $p_{s}{ }^{\alpha_{s}}$ divides

$$
f \cdot \frac{\partial g}{\partial z_{l}}=f \cdot u \cdot \sum_{j=1}^{m} p_{1}{ }^{\alpha_{1}} \cdots \widehat{p_{j}^{\alpha_{j}}} \cdots p_{m}{ }^{\alpha_{m}} \cdot \alpha_{j} \cdot{p_{j}}^{\alpha_{j}-1} \cdot \frac{\partial p_{j}}{\partial z_{l}} .
$$


If $j \neq s$, then the corresponding summand is trivially divisible by $p_{s}{ }^{\alpha_{s}}$. But this implies that $p_{s}{ }^{\alpha_{s}}$ divides

$$
f \cdot u \cdot p_{1}^{\alpha_{1}} \cdots \widehat{p_{s}^{\alpha_{s}}} \cdots p_{m}^{\alpha_{m}} \cdot \alpha_{s} \cdot p_{s}^{\alpha_{s}-1} \cdot \frac{\partial p_{s}}{\partial z_{l}} .
$$

Hence

$$
p_{s} \mid f \cdot p_{1}^{\alpha_{1}} \cdots \widehat{p_{s}^{\alpha_{s}}} \cdots p_{m}^{\alpha_{m}} \cdot \frac{\partial p_{s}}{\partial z_{l}} .
$$

Since $p_{s}$ is prime, it divides one of the factors. Since the primes $p_{1}, \ldots, p_{m}$ are pairwise non-associate, we get for all $l \in\{1, \ldots, n\}$ :

$$
p_{s} \mid f \text { or } p_{s} \mid \frac{\partial p_{s}}{\partial z_{l}}
$$

But since $p_{s}$ is prime and hence $\left(\partial p_{s}\right) /\left(\partial z_{l_{s}}\right) \neq 0$ for some $l_{s} \in\{1, \ldots, n\}$, we get $p_{s} \mid f$. We have shown that every prime factor of $g$ divides $f$. But by reversing the roles of $f$ and $g$ and repeating the same argument, we also get that every prime factor of $f$ divides $g$. We conclude that there exist a unit $v \in \mathbb{C} \backslash\{0\}$ and positive integers $\beta_{1}, \ldots, \beta_{m}$, such that

$$
f=v p_{1}{ }^{\beta_{1}} \cdots p_{m}{ }^{\beta_{m}} .
$$

By assumption we then have for all $l \in\{1, \ldots, n\}$ :

$$
\begin{aligned}
& (\operatorname{deg} g) \cdot u p_{1}{ }^{\alpha_{1}} \cdots p_{m}{ }^{\alpha_{m}} \cdot v \cdot \sum_{j=1}^{m} p_{1}^{\beta_{1}} \cdots \widehat{p_{j} \beta_{j}} \cdots p_{m}{ }^{\beta_{m}} \cdot \beta_{j} \cdot p_{j}{ }^{\beta_{j}-1} \cdot \frac{\partial p_{j}}{\partial z_{l}} \\
& =(\operatorname{deg} f) \cdot v p_{1}{ }^{\beta_{1}} \cdots p_{m}{ }^{\beta_{m}} \cdot u \cdot \sum_{j=1}^{m}{p_{1}}^{\alpha_{1}} \cdots \widehat{p_{j}^{\alpha_{j}}} \cdots p_{m}{ }^{\alpha_{m}} \cdot \alpha_{j} \cdot p_{j}{ }^{\alpha_{j}-1} \cdot \frac{\partial p_{j}}{\partial z_{l}}
\end{aligned}
$$

and hence

$$
0=\sum_{j=1}^{m} p_{1} \cdots \widehat{p_{j}} \cdots p_{m} \cdot \frac{\partial p_{j}}{\partial z_{l}} \cdot\left(\beta_{j}(\operatorname{deg} g)-\alpha_{j}(\operatorname{deg} f)\right)
$$

Considering any $s \in\{1, \ldots, m\}$, we note that $p_{s}$ obviously divides the $j$ th summand for $j \neq s$, and hence

$$
p_{s} \mid p_{1} \cdots \widehat{p_{s}} \cdots p_{m} \cdot \frac{\partial p_{s}}{\partial z_{l}} \cdot\left(\beta_{s}(\operatorname{deg} g)-\alpha_{s}(\operatorname{deg} f)\right) .
$$


Using again that the primes $p_{1}, \ldots, p_{m}$ are pairwise non-associate and considering some $l_{s} \in\{1, \ldots, n\}$ with $\left(\partial p_{s}\right) /\left(\partial z_{l_{s}}\right) \neq 0$, we get

$$
p_{s} \mid\left(\beta_{s}(\operatorname{deg} g)-\alpha_{s}(\operatorname{deg} f)\right)
$$

i.e., $\beta_{s}(\operatorname{deg} g)-\alpha_{s}(\operatorname{deg} f)=0$. So, since $s$ was chosen arbitrarily, we have

$$
\frac{\beta_{j}}{\alpha_{j}}=\frac{\operatorname{deg} f}{\operatorname{deg} g} \text { for all } j \in\{1, \ldots, m\} .
$$

Since $g=h^{M_{g}}$, we can write

$$
h=w p_{1}^{\gamma_{1}} \cdots p_{m}^{\gamma_{m}}
$$

where $w \in \mathbb{C} \backslash\{0\}, w^{M_{g}}=u$, and $\gamma_{j}=\alpha_{j} / M_{g}$ is a positive integer for $j=$ $1, \ldots, m$. Due to the defining properties of $M_{g}$ (see Notation 6.1) we furthermore have $\operatorname{gcd}\left(\gamma_{1}, \ldots, \gamma_{m}\right)=1$, i.e., there exist $d_{1}, \ldots, d_{m} \in \mathbb{Z}$, such that

$$
1=\sum_{j=1}^{m} d_{j} \gamma_{j}
$$

Hence

$$
M_{g} \cdot \frac{\operatorname{deg} f}{\operatorname{deg} g}=\sum_{j=1}^{m} d_{j} \cdot \gamma_{j} \cdot M_{g} \cdot \frac{\operatorname{deg} f}{\operatorname{deg} g}=\sum_{j=1}^{m} d_{j} \cdot \frac{\alpha_{j}}{M_{g}} \cdot M_{g} \cdot \frac{\beta_{j}}{\alpha_{j}}=\sum_{j=1}^{m} d_{j} \beta_{j}
$$

is a (positive) integer, as desired. Finally, we compute

$$
\begin{aligned}
h^{M_{g} \cdot \frac{\operatorname{deg} f}{\operatorname{deg} g}} & =w^{M_{g} \cdot \frac{\operatorname{deg} f}{\operatorname{deg} g}} \cdot \prod_{j=1}^{m}\left(p_{j}^{\gamma_{j}}\right)^{M_{g} \cdot \frac{\beta_{j}}{\alpha_{j}}} \\
& =w^{M_{g} \cdot \frac{\operatorname{deg} f}{\operatorname{deg} g}} \cdot \prod_{j=1}^{m}\left(p_{j}^{\frac{\alpha_{j}}{M_{g}}}\right)^{M_{g} \cdot \frac{\beta_{j}}{\alpha_{j}}} \\
& =w^{M_{g} \cdot \frac{\operatorname{deg} f}{\operatorname{deg} g}} p_{1}^{\beta_{1}} \cdots p_{m}^{\beta_{m}} \\
& =\frac{w^{M_{g} \cdot \frac{\operatorname{deg} f}{\operatorname{deg} g}}}{v} \cdot f,
\end{aligned}
$$

and the claim follows.

Armed with Lemma 6.2, we can provide a proof for Theorem 2.3. 
Proof of Theorem 2.3 As in Sect. 3 we write

$$
P(z)=\sum_{\beta: 1 \leq|\beta| \leq 2 k-1} \bar{z}^{\beta} P_{\beta}(z) .
$$

We then apply Lemma 3.2 and get for all $\beta \in\left(\mathbb{Z}_{\geq 0}\right)^{n}$ with $1 \leq|\beta| \leq 2 k-1$ and for all $j, l \in\{1, \ldots, n\}$ :

$$
\frac{\partial P_{\beta}}{\partial z_{l}} \frac{\partial G}{\partial z_{j}}=\frac{\partial P_{\beta}}{\partial z_{j}} \frac{\partial G}{\partial z_{l}} \text { on } \mathbb{C}^{n} .
$$

Writing $G=G(0)+q+R$, where $q: \mathbb{C}^{n} \rightarrow \mathbb{C}$ is a non-constant, homogeneous, holomorphic polynomial and $R: \mathbb{C}^{n} \rightarrow \mathbb{C}$ is a holomorphic function whose Taylor series at 0 does not involve any terms of degree $\leq \operatorname{deg} q$, we get, owing to the fact that the $P_{\beta}$ are homogeneous:

$$
\frac{\partial P_{\beta}}{\partial z_{l}} \frac{\partial q}{\partial z_{j}}=\frac{\partial P_{\beta}}{\partial z_{j}} \frac{\partial q}{\partial z_{l}} \text { on } \mathbb{C}^{n}
$$

for all $\beta \in\left(\mathbb{Z}_{\geq 0}\right)^{n}$ with $1 \leq|\beta| \leq 2 k-1$ and for all $j, l \in\{1, \ldots, n\}$. By multiplying with $z_{j}$ and then summing over $j$, we get, using that both $P_{\beta}$ and $q$ are homogeneous:

$$
\left(\operatorname{deg} P_{\beta}\right) \cdot P_{\beta} \cdot \frac{\partial q}{\partial z_{l}}=(\operatorname{deg} q) \cdot q \cdot \frac{\partial P_{\beta}}{\partial z_{l}} \text { on } \mathbb{C}^{n}
$$

for all $\beta, l$. Let $h: \mathbb{C}^{n} \rightarrow \mathbb{C}$ be any homogeneous, holomorphic polynomial with $q=h^{M_{q}}$ (see Notation 6.1). Lemma 6.2 then implies that, for a given $\beta$, the following is true:

- if $M_{q} \cdot \frac{\operatorname{deg} P_{\beta}}{\operatorname{deg} q}=M_{q} \cdot \frac{\operatorname{deg} P_{\beta}}{M_{q} \cdot \operatorname{deg} h}=\frac{2 k-|\beta|}{\operatorname{deg} h}$ is not a positive integer, then $P_{\beta} \equiv 0$,

- if $\frac{2 k-|\beta|}{\operatorname{deg} h}$ is a positive integer, then there exists a $c_{\beta} \in \mathbb{C}$, such that

$$
P_{\beta}=c_{\beta} \cdot h^{M_{q} \cdot \frac{\operatorname{deg} P_{\beta}}{\operatorname{deg} q}}=c_{\beta} \cdot h^{\frac{2 k-|\beta|}{\operatorname{deg} h}}
$$

(if $P_{\beta} \equiv 0$, take $c_{\beta}=0$, otherwise apply Lemma 6.2).

Since $P \not \equiv 0$, there exists a positive integer $L$, such that

$$
\{1, \ldots, L\}=\left\{\frac{2 k-|\beta|}{\operatorname{deg} h}: \beta \in\left(\mathbb{Z}_{\geq 0}\right)^{n}, 1 \leq|\beta| \leq 2 k-1, \frac{2 k-|\beta|}{\operatorname{deg} h} \in \mathbb{Z}_{>0}\right\} .
$$

Hence we can write on $\mathbb{C}^{n}$ :

$$
P(z)=\sum_{l=1}^{L} \sum_{\beta:|\beta|=2 k-l \cdot \operatorname{deg} h} \bar{z}^{\beta} \cdot c_{\beta} \cdot(h(z))^{l}
$$




$$
\begin{aligned}
& =\sum_{l=1}^{L}(h(z))^{l} \cdot \sum_{\beta:|\beta|=2 k-l \cdot \operatorname{deg} h} c_{\beta} \cdot \bar{z}^{\beta} \\
& =\sum_{l=1}^{L} \overline{h(z)}{ }^{l} \cdot \sum_{\beta:|\beta|=2 k-l \cdot \operatorname{deg} h} \overline{c_{\beta}} \cdot z^{\beta},
\end{aligned}
$$

where the last equality is due to the fact that $P$ is real-valued. Since $h^{l} \not \equiv 0$, we can find an $\alpha_{l} \in\left(\mathbb{Z}_{\geq 0}\right)^{n}$ with $\left|\alpha_{l}\right|=l \cdot \operatorname{deg} h$, such that $z^{\alpha_{l}}$ appears with coefficient $\gamma_{l} \neq 0$ in the Taylor expansion of $h^{l}$ at 0 (note that $\alpha_{l}$ is not uniquely determined in general). Recalling that

$$
P(z)=\sum_{\beta: 1 \leq|\beta| \leq 2 k-1} \bar{z}^{\beta} P_{\beta}(z),
$$

we see that we necessarily have (recall that $h$ is homogeneous):

$$
P_{\alpha_{l}}(z)=\overline{\gamma_{l}} \cdot \sum_{\beta:|\beta|=2 k-l \cdot \operatorname{deg} h} \overline{c_{\beta}} \cdot z^{\beta},
$$

and hence

$$
P(z)=\sum_{l=1}^{L} \frac{h_{h} l}{l} \cdot \frac{1}{\overline{\gamma_{l}}} \cdot P_{\alpha_{l}}(z) .
$$

Assume for the sake of a contradiction that $2 k /(\operatorname{deg} h)$ is not an integer. Then we have for all $l=1, \ldots, L$ that

$$
\frac{2 k-\left|\alpha_{l}\right|}{\operatorname{deg} h}=\frac{2 k}{\operatorname{deg} h}-l \notin \mathbb{Z},
$$

which implies that $P_{\alpha_{l}} \equiv 0$ (see above). But then $P \equiv 0$ and we arrive at the desired contradiction. Hence $2 k /(\operatorname{deg} h) \in \mathbb{Z}$. But then we have that $\frac{2 k-\left|\alpha_{l}\right|}{\operatorname{deg} h}$ is a positive integer for $l=1, \ldots, L$, which, using the above, implies that

$$
P_{\alpha_{l}}=c_{\alpha_{l}} \cdot h^{\frac{2 k-\left|\alpha_{l}\right|}{\operatorname{deg} h}}=c_{\alpha_{l}} \cdot h^{\frac{2 k}{\operatorname{deg} h}-l}
$$

We now define $s: \mathbb{C} \rightarrow \mathbb{R}$

$$
\tau \mapsto \sum_{l=1}^{L} \frac{c_{\alpha_{l}}}{\overline{\gamma_{l}}} \cdot \bar{\tau}^{l} \cdot \tau^{\frac{2 k}{\operatorname{deg} h}-l}
$$

Note that $s$ is indeed real-valued. It is now easy to see that $s$ and $h$ have all the desired properties. 


\section{Proof of Theorem 2.4 and Corollary 2.5}

In this section we will provide a proof for Theorem 2.4. We will not provide a separate proof for Corollary 2.5, since it will be obvious from the proof of Theorem 2.4.

Proof of Theorem 2.4 We adapt the notation from Sect. 3. In particular we write

$$
P(z)=\sum_{\beta: 1 \leq|\beta| \leq 2 k-1} \bar{z}^{\beta} P_{\beta}(z)=\sum_{\beta \in B} \bar{z}^{\beta} P_{\beta}(z),
$$

where $B=\left\{\beta \in\left(\mathbb{Z}_{\geq 0}\right)^{n}: 1 \leq|\beta| \leq 2 k-1\right.$ and $\left.P_{\beta} \not \equiv 0\right\}$. By assumption on $P$ we find, for every $\beta \in \bar{B}$, a holomorphic polynomial $q_{\beta}: \mathbb{C}^{n-l} \rightarrow \mathbb{C}$, homogeneous of degree $2 k-\left(2 d_{1}+\cdots+2 d_{l}+\beta_{l+1}+\cdots+\beta_{n}\right)$, such that

$$
P_{\beta}\left(z_{1}, \ldots, z_{n}\right)=z_{1}^{2 d_{1}-\beta_{1}} \cdots z_{l}^{2 d_{l}-\beta_{l}} q_{\beta}\left(z_{l+1}, \ldots, z_{n}\right) .
$$

For ease of notation we write $m=n-l$ and $\left(w_{1}, \ldots, w_{m}\right)=\left(z_{l+1}, \ldots, z_{n}\right)$. We will switch back and forth between notations whenever convenient. By Lemma 3.5, away from the coordinate hyperplanes, $P$ is pluriharmonic along the level sets of $G: \mathbb{C}^{n} \rightarrow \mathbb{C}^{m}$

$$
G\left(z_{1}, \ldots, z_{l}, w_{1}, \ldots, w_{m}\right)=z_{1}{ }^{d_{1}} \ldots z_{l}{ }^{d_{l}} \cdot\left(w_{1}{ }^{k-D}, \ldots, w_{m}{ }^{k-D}\right)
$$

Lemma 3.2 then gives that the following holds on $\mathbb{C}^{n}$ for all $\beta \in B$ and for all $v \in\{1, \ldots, n\}:$

$$
\operatorname{det}\left(\begin{array}{ccc}
\frac{\partial G_{1}}{\partial w_{1}} & \cdots & \frac{\partial G_{1}}{\partial w_{m}} \\
\vdots & \ddots & \vdots \\
\frac{\partial G_{m}}{\partial w_{1}} & \cdots & \frac{\partial G_{m}}{\partial w_{m}}
\end{array}\right) \cdot \frac{\partial P_{\beta}}{\partial z_{v}}=\sum_{j=1}^{m} \operatorname{det}\left(\begin{array}{ccc}
\frac{\partial G_{1}}{\partial w_{1}} & \cdots & \frac{\partial G_{1}}{\partial w_{m}} \\
\vdots & \ddots & \vdots \\
\frac{\partial G_{j-1}}{\partial w_{1}} & \cdots & \frac{\partial G_{j-1}}{\partial w_{m}} \\
\frac{\partial P_{\beta}}{\partial w_{1}} & \cdots & \frac{\partial P_{\beta}}{\partial w_{m}} \\
\frac{\partial G_{j+1}}{\partial w_{1}} & \cdots & \frac{\partial G_{j+1}}{\partial w_{m}} \\
\vdots & \ddots & \vdots \\
\frac{\partial G_{m}}{\partial w_{1}} & \cdots & \frac{\partial G_{m}}{\partial w_{m}}
\end{array}\right) \cdot \frac{\partial G_{j}}{\partial z_{v}}
$$

A calculation then shows that the following holds on $\mathbb{C}^{n}$ for all $\beta \in B, v \in\{1, \ldots, l\}$ :

$$
(k-D)\left(2 d_{v}-\beta_{v}\right) q_{\beta}\left(w_{1}, \ldots, w_{m}\right)=d_{v} \sum_{j=1}^{m} w_{j} \frac{\partial q_{\beta}}{\partial w_{j}}\left(w_{1}, \ldots, w_{m}\right)
$$

but since $q_{\beta} \not \equiv 0$ is homogeneous, this simplifies to

$$
\begin{gathered}
(k-D)\left(2 d_{v}-\beta_{v}\right) q_{\beta}\left(w_{1}, \ldots, w_{m}\right) \\
=d_{v} \cdot\left(2 k-\left(2 d_{1}+\cdots+2 d_{l}+\beta_{l+1}+\cdots+\beta_{n}\right)\right) \cdot q_{\beta}\left(w_{1}, \ldots, w_{m}\right) .
\end{gathered}
$$


For $\beta \in B$ we have $q_{\beta} \not \equiv 0$, so we get for all $\beta \in B, v \in\{1, \ldots, l\}$ :

$$
\begin{aligned}
(k-D)\left(2 d_{v}-\beta_{v}\right) & =d_{v} \cdot\left(2 k-\left(2 d_{1}+\cdots+2 d_{l}+\beta_{l+1}+\cdots+\beta_{n}\right)\right) \\
& =d_{v} \cdot M_{\beta},
\end{aligned}
$$

where $M_{\beta}$ is defined in the obvious way. Since $d=\operatorname{gcd}\left(d_{1}, \ldots, d_{l}, k\right)$, there exist $c, c_{1}, \ldots, c_{l} \in \mathbb{Z}$, such that $d=c \cdot(k-D)+\sum_{\nu=1}^{l} c_{v} \cdot d_{\nu}$. But then we have for all $\beta \in B$ :

$$
\frac{d \cdot M_{\beta}}{k-D}=c \cdot M_{\beta}+\sum_{\nu=1}^{l} c_{\nu} d_{\nu} \frac{M_{\beta}}{k-D}=c \cdot M_{\beta}+\sum_{\nu=1}^{l} c_{\nu}\left(2 d \nu-\beta_{\nu}\right)
$$

so $d \cdot M_{\beta} /(k-D)$ is an integer for all $\beta \in B$. If $M_{\beta}$ was 0 for some $\beta \in B$, then $2 d_{v}-\beta_{v}$ would be 0 for $v \in\{1, \ldots, l\}$, implying that $|\beta|=2 k$, in contradiction to $\beta \in B$. Hence $d \cdot M_{\beta} /(k-D)$ is a positive integer for all $\beta \in B$. This implies that for all $\beta \in B$ we have

$$
P_{\beta}\left(z_{1}, \ldots, z_{n}\right)=\left(z_{1}^{\frac{d_{1}}{d}} \cdots z_{l}^{\frac{d_{l}}{d}}\right)^{\frac{d \cdot M_{\beta}}{k-D}} \cdot q_{\beta}\left(z_{l+1}, \ldots, z_{n}\right),
$$

where all occurring exponents are positive integers. If $\beta \in B$, then $M_{\beta} \leq 2(k-D)$. If this was an equality, then, similarly to above, we would get $|\beta|=0$, in contradiction to $\beta \in B$. Hence we have for all $\beta \in B$ :

$$
\frac{d \cdot M_{\beta}}{k-D}, 2 d-\frac{d \cdot M_{\beta}}{k-D} \in\{1,2, \ldots, 2 d-1\}
$$

For $\beta \in B, v \in\{1, \ldots, l\}$ we have

$$
\beta_{v}=2 d_{v}-\frac{d_{v} \cdot M_{\beta}}{k-D}=\frac{d_{v}}{d} \cdot\left(2 d-\frac{d \cdot M_{\beta}}{k-D}\right),
$$

and both factors in the latter equality are positive integers. We now calculate, noting that all occurring exponents are positive integers:

$$
\begin{aligned}
P(z)= & \sum_{\beta \in B} \bar{z}^{\beta} P_{\beta}(z) \\
= & \sum_{\beta \in B}\left(z_{1}^{\frac{d_{1}}{d}} \cdots z_{l}^{\frac{d_{l}}{d}}\right)^{\frac{d \cdot M_{\beta}}{k-D}} \cdot{\overline{\left(z_{1} \frac{d_{1}}{d} \cdots z_{l} \frac{d_{l}}{d}\right.}}^{2 d-\frac{d \cdot M_{\beta}}{k-D}} \\
& \cdot \bar{z}_{l+1}^{\beta_{l+1}} \cdots{\overline{z_{n}}}^{\beta_{n}} q_{\beta}\left(z_{l+1}, \ldots, z_{n}\right) \\
= & \sum_{j=1}^{2 d-1}\left(z_{1}^{\frac{d_{1}}{d}} \cdots z_{l}^{\frac{d_{l}}{d}}\right)^{j} \cdot{\overline{\left(z_{1}^{\frac{d_{1}}{d}} \cdots z_{l}^{\frac{d_{l}}{d}}\right)}}^{2 d-j}
\end{aligned}
$$




$$
\sum_{\beta \in B: \frac{d \cdot M_{\beta}}{k-D}=j} \overline{z_{l+1}} \beta_{l+1} \ldots \overline{z_{n}} \beta_{n} q_{\beta}\left(z_{l+1}, \ldots, z_{n}\right)
$$

Now if $\beta \in B$ and $j \in\{1, \ldots, 2 d-1\}$ satisfy $d \cdot M_{\beta} /(k-D)=j$, then every term occurring in $\overline{z_{l+1}} \beta_{l+1} \ldots{\overline{z_{n}}}^{\beta_{n}} q_{\beta}\left(z_{l+1}, \ldots, z_{n}\right)$ has holomorphic degree $M_{\beta}=$ $j \cdot(k-D) / d$ and anti-holomorphic degree $\beta_{l+1}+\cdots+\beta_{n}=(2 d-j) \cdot(k-D) / d$. This implies that the polynomial $Q: \mathbb{C}^{m} \rightarrow \mathbb{R}$,

$$
Q\left(w_{1}, \ldots, w_{m}\right)=\sum_{j=1}^{2 d-1} \sum_{\beta \in B: \frac{d \cdot M_{\beta}}{k-D}=j}{\overline{w_{1}}}^{\beta_{1+l}} \ldots{\overline{w_{m}}}^{\beta_{m+l}} q_{\beta}\left(w_{1}, \ldots, w_{m}\right),
$$

has the property that both the holomorphic and the anti-holomorphic degree of every term appearing in $Q$ are divisible by the integer $(k-D) / d$. Furthermore $Q\left(w_{1}, \ldots, w_{m}\right)=P\left(1, \ldots, 1, w_{1}, \ldots, w_{m}\right)$, so $Q$ is (indeed real-valued and) plurisubharmonic. It is now easy to see that $Q$ has all the other desired properties.

Funding Open access funding provided by NTNU Norwegian University of Science and Technology (incl St. Olavs Hospital - Trondheim University Hospital).

Open Access This article is licensed under a Creative Commons Attribution 4.0 International License, which permits use, sharing, adaptation, distribution and reproduction in any medium or format, as long as you give appropriate credit to the original author(s) and the source, provide a link to the Creative Commons licence, and indicate if changes were made. The images or other third party material in this article are included in the article's Creative Commons licence, unless indicated otherwise in a credit line to the material. If material is not included in the article's Creative Commons licence and your intended use is not permitted by statutory regulation or exceeds the permitted use, you will need to obtain permission directly from the copyright holder. To view a copy of this licence, visit http://creativecommons.org/licenses/by/4.0/.

\section{References}

1. Bedford, E., Fornæss, J.E.: A construction of peak functions on weakly pseudoconvex domains. Ann. Math. (2) 107(3), 555-568 (1978)

2. Bharali, G.: Model pseudoconvex domains and bumping. Int. Math. Res. Not. IMRN 21, 4924-4965 (2012)

3. Bharali, G., Stensønes, B.: Plurisubharmonic polynomials and bumping. Math. Z. 261(1), 39-63 (2009)

4. Fornæss, J.E.: Sup-norm estimates for $\bar{\partial}$ in $\mathbf{C}^{2}$. Ann. Math. (2) 123(2), 335-345 (1986)

5. Fornæss, J.E., Sibony, N.: Construction of P.S.H. functions on weakly pseudoconvex domains. Duke Math. J. 58(3), 633-655 (1989)

6. Fornæss, J.E., Stensønes, B.: Maximally tangent complex curves for germs of finite type $\mathcal{C}^{\infty}$ pseudoconvex domains in $\mathbb{C}^{3}$. Math. Ann. 347(4), 979-991 (2010)

7. Grundmeier, D., Simon, L., Stensønes, B.: Sup-norm estimates for $\bar{\partial}$ in $\mathbb{C}^{3}$ (2020). arXiv:1909.04080

8. Noell, A.: Peak Functions for Pseudoconvex Domains in $\mathbf{C}^{n}$, Several Complex Variables (Stockholm, 1987/1988), vol. 38, pp. 529-541. Princeton University Press, Princeton (1993)

9. Range, R.M.: Integral kernels and Hölder estimates for $\bar{\partial}$ on pseudoconvex domains of finite type in $\mathbf{C}^{2}$. Math. Ann. 288(1), 63-74 (1990)

Publisher's Note Springer Nature remains neutral with regard to jurisdictional claims in published maps and institutional affiliations. 